H. Morikawa

Nagoya Math. J.

Vol. 77 (1980), 75-87

\title{
ON DIFFERENTIAL INVARIANTS OF HOLOMORPHIC PROJECTIVE CURVES
}

\author{
HISASI MORIKAWA
}

\section{Canonical forms}

Let $\left(\varphi_{1}(u), \cdots, \varphi_{n}(u)\right)$ be a system of holomorphic functions whose Wronskian does not vanish at origin, where holomorphic functions mean functions holomorphic around origin.

A variable transformation

$$
(u, y) \mapsto(u(z), \lambda(z) y)
$$

induces a map

$$
\begin{aligned}
\left(\varphi_{1}(u), \cdots, \varphi_{n}(u)\right) & \mapsto\left(\phi_{1}(z), \cdots, \phi_{n}(z)\right) \\
= & \left(\lambda(z) \varphi_{1}(u(z)), \cdots, \lambda(z) \varphi_{n}(u(z))\right),
\end{aligned}
$$

where $\frac{d u}{d z}(0) \neq 0$ and $\lambda(0) \neq 0$.

We associate linear differential operators of rank $n$ which is a projective invariant of a holomorphic curve ${ }^{1)}: u \mapsto\left(\varphi_{1}(u), \cdots, \varphi_{n}(u)\right)$ in $\boldsymbol{P}^{n-1}$.

$$
\begin{aligned}
& L_{n}(p \mid u, y)=\sum_{l=0}^{n}\left(\begin{array}{l}
n \\
l
\end{array}\right) p_{l}(u)\left(\frac{d}{d u}\right)^{n-l} y
\end{aligned}
$$

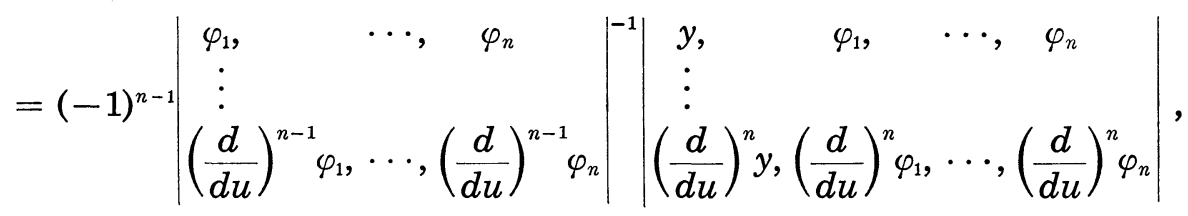

$$
\begin{aligned}
& L_{n}(q \mid z, y)=\sum_{l=0}^{n}\left(\begin{array}{l}
n \\
l
\end{array}\right) q_{\imath}\left(z\left(\frac{d}{d z}\right)\right)^{n-l} y
\end{aligned}
$$

Received December 6, 1978.

1) We mean holomorphic maps by holomorphic curves. 


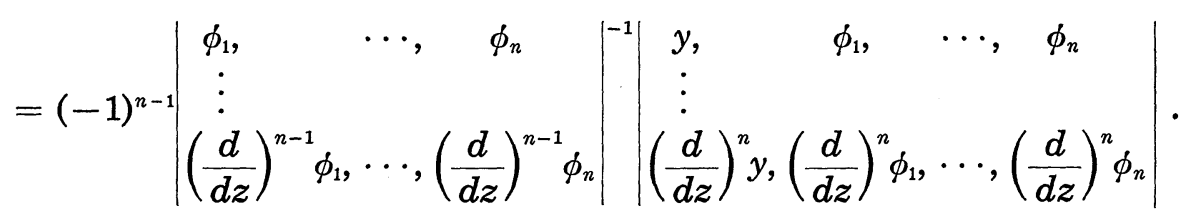

The variable transformation $(u, y) \mapsto(u(z), \lambda(z) y)$ induces a transformation:

$$
\begin{aligned}
& L_{n}(p \mid u, y) \mapsto L_{n}(q \mid z, y) \\
&=\lambda(z)^{-1}\left(\frac{d u}{d z}\right)^{-n} \sum\left(\frac{n}{l}\right) p_{l}(u(z))\left(\frac{d}{d u(z)}\right)^{n-l}(\lambda(z) y) .
\end{aligned}
$$

Definition (Lagurre-Forsyth). A linear differential operator $L_{n}(Q \mid z, y)$ is called to be canonical, if

$$
Q_{1} \equiv Q_{2} \equiv 0
$$

We call $z$ a canonical independent variable of a canonical form $L_{n}(Q \mid z, y)$.

TheOREM 1 (Forsyth) $)^{2}$. For each $L_{n}(p \mid u, z)$ there exists a variable transformation

$$
(u, y) \mapsto(u(z), \lambda(z) y)
$$

such that $L_{n}(p \mid u, z)$ is transformed to a canonical form. Moreover a variable transformation maps a canonical form to a canonical form, if and only if

$$
u(z)=\frac{\alpha z+\beta}{\gamma z+\delta}, \quad \lambda(z)=\frac{c y}{(\gamma z+\delta)^{n-1}} \quad\left(\left(\begin{array}{l}
\delta \beta \\
\gamma \alpha
\end{array}\right) \in S L(2, C), c \in C-\{0\}\right) .
$$

Forsyth's theorem means that for each holomorphic projective curve:

$$
u \mapsto\left(\varphi_{1}(u), \cdots, \varphi_{n}(u)\right) \in \boldsymbol{P}^{n-1}
$$

we may associate a canonical variable and a canonical form which are unique up to Möbius transformations.

\section{Differential invariants}

Similarly as classical invariant theory, we define differential invariants of $L_{n}(P \mid u, y)$ first for generic coefficients $P_{i}(u)(1 \leq i \leq n)$, and then specialization

2) See Theorem 6.1 and 6.2, Chap 6 [1], or Theorem 2.4, Chap 2 [2]. 


$$
\left(\frac{d}{d u}\right)^{l} P_{i}(u) \mapsto\left(\frac{d}{d u}\right)^{l} p_{i}(u) \quad(1 \leq i \leq n ; 0 \leq l<\infty)
$$

gives the definition for $L_{n}(p \mid u, y)$.

Definition. A differential invariant of weight $p$ of $L_{n}(P \mid u, y)$ (with generic coefficients) is a polynomial

$$
\Phi\left(\cdots,\left(\frac{d}{d z}\right)^{l} P_{j}(u), \cdots\right)
$$

such that

$$
\Phi\left(\cdots,\left(\frac{d}{d u}\right)^{l} P_{j}(u), \cdots\right)(d u)^{p}
$$

is invariant for every variable transformation

$$
(u, y) \mapsto(u(z), \lambda(z) y)
$$

where

$$
\frac{d u(0)}{d z} \neq 0, \quad \lambda(0) \neq 0
$$

Forsyth gave the following fundamental system of differential invariants of a canonical form

$$
\begin{gathered}
L_{n}(Q \mid z, y)=\left(\frac{d}{d z}\right)^{n} y+\sum_{l=3}^{n}\left(\begin{array}{c}
n \\
l
\end{array}\right) Q_{l}(z)\left(\frac{d}{d z}\right)^{n-l} y ; \\
\left(\theta_{3}(z), \cdots, \theta_{n}(z)\right), \\
\theta_{p}(z)=\frac{1}{2} \sum \frac{(-1)^{s}(p-2) ! p !(2 p-s-2) !}{(p-s-1) !(p-s) !(2 p-3) ! s !}\left(\frac{d}{d z}\right)^{s} Q_{p-s}(z) \quad(3 \leq p \leq n),
\end{gathered}
$$

where weight of $\theta_{p}(z)$ is $p$.

For a complex number $w(\neq 0,1,2, \cdots)$ we denote

$$
\left(\begin{array}{l}
w \\
l
\end{array}\right)=\frac{w(w-1) \cdots(w-l+1)}{l 1}
$$

Let $w_{1}, \cdots, w_{n}$ be complex numbers $(\neq 0,1,2, \cdots)$,

$$
\xi_{1}=\left(\xi_{1}^{(0)}, \xi_{1}^{(1)}, \xi_{1}^{(2)}, \cdots\right), \cdots, \xi_{n}=\left(\xi_{n}^{(0)}, \xi_{n}^{(1)}, \xi_{n}^{(2)}, \cdots\right)
$$

be variable vectors of infinite length and denote 


$$
f_{j}\left(\xi_{j} \mid z\right)=\sum_{l=0}^{\infty}\left(\begin{array}{c}
w_{j} \\
l
\end{array}\right) \xi_{j}^{(l)} z^{l} \quad(1 \leq j \leq n) .
$$

Germ of $S L(2, C)$ at $\left(\begin{array}{ll}1 & 0 \\ 0 & 1\end{array}\right)$ acts on $f_{j}\left(\xi_{j} \mid z\right)$ as follows

$$
f_{j}\left(\left(\begin{array}{c}
\delta \beta \\
\gamma \alpha
\end{array}\right) \xi_{j} \mid z\right)=(\gamma z+\delta)^{w_{j}} f_{j}\left(\xi_{j} \mid \frac{\alpha z+\beta}{\gamma z+\delta}\right) \quad(1 \leq j \leq n),
$$

where $(\gamma z+\delta)^{w_{j}}=\delta^{w_{j}} \sum_{l=0}^{\infty}\left(\begin{array}{c}w_{j} \\ l\end{array}\right)\left(\delta^{-1} \gamma z\right)^{l}$.

Definition. A formal power series $F(\xi ; z)$ with coefficients in $C[\xi]$ is called a covariant of index $u$ (a complex number), if

$$
\begin{gathered}
F(\xi ; z)=\sum_{l=0}^{\infty}\left(\begin{array}{l}
u \\
l
\end{array}\right) c_{l}(\xi) z^{l}, \\
F\left(\left(\begin{array}{l}
\delta \beta \\
\gamma \alpha
\end{array}\right) \xi ; z\right)=(\gamma z+\delta)^{u} F\left(\xi ; \frac{\alpha z+\beta}{\gamma z+\delta}\right), \quad\left(\left(\begin{array}{l}
\delta \beta \\
\gamma \alpha
\end{array}\right) \in \text { Germ of } S L(2, C)\right) .
\end{gathered}
$$

Definition. A polynomial $\varphi(\xi)$ is called a semi-invariant of index $u$, if

$$
\sum_{j=1}^{n} \sum_{l=0}^{\infty} l \xi_{j}^{(l-1)} \frac{\partial}{\partial \xi_{j}^{(l)}} \varphi(\xi)=0
$$

and

$$
\sum_{j=1}^{n} \sum_{l=0}^{\infty}\left(w_{j}-2 l\right) \xi_{j}^{(l)} \frac{\partial}{\partial \xi_{j}^{(l)}} \varphi(\xi)=u \varphi(\xi)
$$

THEOREM $2^{3)}$. The following three conditions are equivalent;

i) $F(\xi ; z)$ is a covariant of index $u$,

ii) $F(\xi ; z)=\exp (z \Delta) \varphi(\xi)$ with a semi-invariant of $\varphi(\xi)$ index $u$, where

$$
\Delta=\sum_{j=1}^{n} \sum_{l=0}^{\infty}\left(w_{j}-l\right) \xi_{j}^{(l+1)} \frac{\partial}{\partial \xi_{j}^{(l)}},
$$

iii) $\quad F(\xi ; z)=\varphi\left(\cdots, \frac{(d / d z)^{l} f_{j}\left(\xi_{j} \mid z\right)}{w_{j}\left(w_{j}-1\right) \cdots\left(w_{j}-l+1\right)}, \cdots\right)$ with a semi-invariant $\varphi\left(\cdots, \xi_{j}^{(l)}, \cdots\right)$ of index $u$.

THEOREM $3^{4)}$. [2].

3) See Theorem 5.1 (Robert's Theorem), Chap. 5 [1], or Theorem 1.3, Chap 1

4) See Theorem 6.5, Chap. 6 [1], or Theorem 2.6, Chap 2 [2]. 


$$
\begin{aligned}
& \left\{\text { Differential invariants of } L_{n}(Q \mid z, y)\right\} \\
& \quad=\left\{\text { covariants of } \theta_{3}(z), \cdots, \theta_{n}(z)\right\},
\end{aligned}
$$

where $\theta_{p}(z)=\sum_{l=0}^{\infty}\left(\begin{array}{c}-2 p \\ l\end{array}\right) \eta_{p}^{(l)} z^{l}$ and $\left(w_{3}, \cdots, w_{n}\right)=(-6,-8, \cdots,-2 n)$.

\section{Defining differential equations of moduli}

Two projective holomorphic curves in $\boldsymbol{P}^{n-1}$

$$
C_{\varphi}: u \rightarrow\left(\varphi_{1}(u), \cdots, \varphi_{n}(u)\right)
$$

and

$$
C_{\phi}: z \rightarrow\left(\phi_{1}(z), \cdots, \phi_{n}(z)\right)
$$

are called to be equivalent, if a projective transformation in $\boldsymbol{P}^{n-1}$ and a variable transformation $(u, y) \rightarrow(u(z), \lambda(z) y)$ map $C_{\varphi}$ to $C_{\phi}$.

By virtue of Forsyth's theorem equivalence classes of projective holomorphic curves correspond bijectively to equivalence classes of canonical forms with respect to transformations

$$
(z, y) \rightarrow\left(\frac{\alpha z+\beta}{\gamma z+\delta}, \frac{c y}{(\gamma z+\delta)^{n-1}}\right) .
$$

Here we shall give the moduli spaces of projective holomorphic curves in $\boldsymbol{P}^{n-1}$ and stable projective holomorphic curves by means of non-linear differential equations. The answer is not so difficult, that is a consequence of properties of Schwarzian derivatives.

Schwarzian

$$
\{z, \tau\}=\frac{\frac{d^{3} z}{d \tau^{3}}}{\frac{d^{2} z}{d \tau^{2}}}-\frac{3}{2}\left(\frac{\frac{d^{2} z}{d \tau^{2}}}{\frac{d z}{d \tau}}\right)^{2}
$$

is naturally generalized to pairs of differential forms as follows

$$
\left\{(d f)^{m}, d g\right\}=\{d f, d g\}=\{f, g\}(d g)^{2} .
$$

LEMma 1 . If we put 


$$
\chi_{j}(z)=\left|\begin{array}{cc}
\theta_{j}(z) & \frac{-1}{2 j} \frac{d \theta_{j}(z)}{d z} \\
\frac{-1}{2 j} \frac{d \theta_{j}(z)}{d z} & \frac{1}{2 j(2 j+1)} \frac{d^{2} \theta_{j}(z)}{d z^{2}}
\end{array}\right|,
$$

we have

$$
\chi_{j}(z)(d z)^{2 j+2}=\frac{\left(\theta_{j}(z)(d z)^{j}\right)^{2}}{2(2 j+1)}\left\{\theta_{j}(z)(d z)^{j}, d z\right\} .
$$

Proof. Putting $\theta_{j}(z)(d z)^{j}=(d f)^{j}$, we have

$$
\begin{aligned}
\chi_{j}(z)(d z)^{2 j+2} & =\left(\frac{(d f / d z)^{j}}{2 j(2 j+1)}\left(\frac{d}{d z}\right)^{2}\left(\frac{d f}{d z}\right)^{j}-\frac{1}{(2 j)^{2}}\left(\frac{d}{d z}\left(\frac{d f}{d z}\right)^{j}\right)^{2}\right)(d z)^{2 j+2} \\
& =\frac{1}{2(2 j+1)}\left(\frac{d f}{d z}\right)^{2 j}\{f, z\} d z^{2 j+2} .
\end{aligned}
$$

LEMMA 2. Let $\theta_{j}(u)(d u)^{j}, \chi_{j}(u)(d u)^{2 j+2}(3 \leq j \leq n)$ be holomorphic differential forms around origin. Then there exists a variable $z$ such that

$$
\chi_{j}(u)(d u)^{2 j+2}=\frac{\left(\theta_{j}(u)(d u)^{j}\right)^{2}}{2(2 j+1)}\left\{\theta_{\jmath}(u)(d u)^{\jmath}, d z\right\} \quad(3 \leq j \leq n),
$$

if and only if

$$
\begin{aligned}
2(2 j+1) \chi_{j}(u) \theta_{k}(u)^{2}-2(2 k+1) \chi_{k}(u) \theta_{j}(u)^{2} \\
=\frac{1}{j} \theta_{j}(u) \theta_{k}(u)^{2} \frac{d^{2} \theta_{j}(u)}{d u^{2}}-\frac{1}{k} \theta_{k}(u) \theta_{j}(u)^{2} \frac{d^{2} \theta_{k}(u)}{d u^{2}} \\
\quad-\frac{2 j+1}{2 j^{2}} \theta_{k}(u)^{2}\left(\frac{d \theta_{j}(u)}{d u}\right)^{2}+\frac{2 k+1}{2 k^{2}} \theta_{j}(u)^{2}\left(\frac{d \theta_{k}(u)}{d u}\right)^{2} \\
(3 \leq j<k \leq n) .
\end{aligned}
$$

If $\left(\theta_{3}(u)(d u)^{3}, \cdots, \theta_{n}(u)(d u)^{n} \neq(0, \cdots, 0)\right.$, then the variable $z$ is uniquely determined up to Möbius transformations.

Proof. If $\theta_{j}(u), \theta_{k}(u) \not \equiv 0$, then from properties of Schwarzian it follows.

$$
\begin{aligned}
&\left\{\theta_{j}(u)(d u)^{j}, d z\right\}-\left\{\theta_{k}(u)(d u)^{k}, d z\right\} \\
&=\left\{\theta_{j}(u)(d u)^{j}, d u\right\}+\{d u, d z\}-\left\{\theta_{k}(u)(d u)^{k}, d u\right\}-\{d u, d z\} \\
&=\left\{\theta_{j}(u)(d u)^{j}, d u\right\}-\left\{\theta_{k}(u)(d u)^{k}, d u\right\} \\
&=\left(\frac{1}{j} \frac{d^{2} \theta_{j}(u)}{d^{2} u} \theta_{j}(u)^{-1}-\frac{2 j+1}{2 j^{2}}\left(\frac{d \theta_{j}(u)}{d u}\right)^{2} \theta_{j}(u)^{-2}\right)(d u)^{2} \\
&-\left(\frac{1}{k} \frac{d^{2} \theta_{k}(u)}{d^{2} u} \theta_{k}(u)^{-1}-\frac{2 k+1}{2 k^{2}}\left(\frac{d \theta_{k}(u)}{d u}\right)^{2} \theta_{j k}(u)^{-2}\right)(d u)^{2} .
\end{aligned}
$$


For a fixed $j$ the equation on $z$

$$
\left\{\theta_{j}(u)(d u)^{j}, d u\right\}-2(2 j+1) \theta_{j}(u)^{-2} \chi_{j}(u)(d u)^{2}=\{d z, d u\}
$$

is solvable, and

$$
\left\{\theta_{j}(u)(d u)^{j}, d z\right\}=2(2 j+1) \theta_{j}(u)^{-2} \chi_{j}(u)(d u)^{2} .
$$

Hence we can choose a variable $z$ such that

$$
\chi_{j}(u)(d u)^{2 j+2}=\frac{\left(\theta_{j}(u)(d u)^{j}\right)^{2}}{2(2 j+1)}\left\{\theta_{j}(u)(d u)^{j}, d z\right\}
$$

if and only if (2). Assume that $\theta_{j}(u) d u \neq 0$. Then

$$
\left\{\theta_{j}(u)(d u)^{j}, d z\right\}-\left\{\theta_{j}(u)(d u)^{j}, d z^{\prime}\right\}=\left\{d z, d z^{\prime}\right\}=0
$$

and thus $z$ is uniquely determined by $\left\{\theta_{j}(u)(d u)^{j}, d z\right\}$ up to Möbius transformations.

Now we have the next theorem:

TheOREM 4. Equivalence classes of holomorphic projective curves in $\boldsymbol{P}^{n-1}$ correspond bijectively to system of holomorphic differential forms

$$
\left(\theta_{3}(u)(d u)^{3}, \cdots, \theta_{n}(u)(d u)^{n}, \chi_{3}(u)(d u)^{8}, \cdots, \chi_{n}(u)(d u)^{2 n+2}\right)
$$

such that

$$
\begin{aligned}
2(2 j+1) \chi_{j}(u) \theta_{k}(u)^{2}-2(2 k+1) \chi_{k}(u) \theta_{j}(u)^{2} & \\
= & \frac{1}{j} \theta_{j}(u) \theta_{k}(u)^{2} \frac{d^{2} \theta_{3}(u)}{d u^{2}}-\frac{1}{k} \theta_{k}(u) \theta_{j}(u)^{2} \frac{d^{2} \theta_{k}(u)}{d u^{2}} \\
& -\frac{2 j+1}{2 j^{2}} \theta_{k}(u)^{2} \frac{d \theta_{j}(u)^{2}}{d u}+\frac{2 k+1}{2 k^{2}} \theta_{j}(u)^{2}\left(\frac{d_{k}(u)}{d u}\right)^{2} \\
& (3 \leq j<k \leq n) .
\end{aligned}
$$

Let $L_{n}(Q \mid z, y)$ be a canonical form associating with a holomorphic projective curve $C$, and $\left(\theta_{3}(z), \cdots, \theta_{n}(z)\right)$ be the system of fundamental differential. invariants of $L_{n}(Q \mid z, y)$. Then the bijective correspondence is given by

$$
\begin{aligned}
\theta_{j}(u)(d u)^{j} & =\theta_{j}(z)(d z)^{j} \\
\chi_{j}(u)(d u)^{2 j+2} & =\frac{\left(\theta_{j}(z)(d z)^{j}\right)^{2}}{2(2 j+1)}\left\{\theta_{j}(z)(d z)^{j}, d z\right\} \quad(3 \leq j \leq n)
\end{aligned}
$$

Proof. It follows from the following equivalence

$$
L_{n}(Q \mid z, y) \leftrightarrow\left(\theta_{3}(z), \cdots, \theta_{n}(z)\right) \leftrightarrow\left(\theta_{3}(z)(d z)^{3}, \cdots, \theta_{n}(z)(d z)^{n}, z\right) .
$$


TheOREm 5. Denote

$$
\begin{aligned}
& \theta_{j h}(z)=\left|\begin{array}{cc}
\theta_{j}(z) & \theta_{h}(z) \\
\frac{-1}{2 j} \frac{d}{d z} \theta_{j}(z) & \frac{-1}{2 h} \frac{d}{d z} \theta_{h}(z)
\end{array}\right| \quad(3 \leq j<h \leq n), \\
& \chi_{j k}(z)=\left|\begin{array}{cc}
\chi_{j}(z) \\
\frac{-1}{4 j+4} \frac{d}{d z} \chi_{j}(z) & \frac{-1}{2 k} \frac{d}{d z} \theta_{k}(z)
\end{array}\right| \quad(3 \leq j, k \leq n) .
\end{aligned}
$$

Let $\tilde{C}$ be the portrait (equivalence class with respect to independent variable transformations) of the curve

$$
z \mapsto\left(\cdots, \theta_{j}(z), \cdots, \theta_{j h}(z), \cdots, \chi_{j}(z), \cdots, \chi_{j k}(z), \cdots\right)
$$

in weighted projective space with weight system

$$
(\cdots, j, \cdots, j+k+1, \cdots, 2 j+2, \cdots, 2 j+k+3, \cdots) .
$$

Then, if $\left(\cdots, \theta_{j h}, \cdots, \chi_{j k}, \cdots\right) \not \equiv(0, \cdots, 0)$, the correspondence:

$$
\text { [equivalence class of } C] \mapsto \tilde{C}
$$

is bijective. If $\left(\cdots, \theta_{j h}, \cdots, \chi_{j k} \cdots\right) \equiv(0, \cdots, 0)$, then $\tilde{C}$ is a point and $C$ is equivalent to a curve

$$
\left(z^{\lambda_{1}}, z^{\lambda_{1}} \log z, \cdots, z^{\lambda_{1}}(\log z)^{m_{1}}, \cdots, z^{\lambda_{r}}, z^{\lambda_{r}} \log z, \cdots, z^{\lambda_{r}}(\log z)^{m_{r}}\right)
$$

with $\lambda_{1}, \cdots, \lambda_{r} \in C$ and $\sum m_{i}=n$.

Proof. Let $\left(\theta_{3}^{\prime}\left(z^{\prime}\right), \cdots, \theta_{n}^{\prime}\left(z^{\prime}\right)\right)$ be the system of fundamental differential invariants of a canonical form $L_{n}\left(Q^{\prime} \mid z^{\prime}, y\right)$ corresponding to the same curve $\tilde{C}$ in weighted projective space. Then there exists $\mu(z) \not \equiv 0$ such that

$$
\begin{aligned}
\theta_{j}^{\prime}\left(z^{\prime}\right) & =\mu(z)^{j} \theta_{j}(z), \\
\theta_{j k}^{\prime}\left(z^{\prime}\right) & =\mu(z)^{j+k+1} \theta_{j k}(z), \\
\chi_{j}^{\prime}\left(z^{\prime}\right) & =\mu(z)^{2 j+2} \chi_{j}(z), \\
\chi_{j k}^{\prime}\left(z^{\prime}\right) & =\mu(z)^{2 j+k+3} \chi_{j k}(z) .
\end{aligned}
$$

On the other hand

$$
\mu(z)^{j+k+1} \theta_{j k}(z)=\theta_{j k}^{\prime}\left(z^{\prime}\right)=\left|\begin{array}{cc}
\theta_{j}^{\prime}\left(z^{\prime}\right) & \theta_{k}^{\prime}\left(z^{\prime}\right) \\
\frac{-1}{2 j} \frac{d}{d z^{\prime}} \theta_{j}^{\prime}\left(z^{\prime}\right) & \frac{-1}{2 k} \frac{d}{d z^{\prime}} \theta_{k}^{\prime}\left(z^{\prime}\right)
\end{array}\right|
$$




$$
\begin{gathered}
=\mu(z)^{j+k} \frac{d z}{d z^{\prime}} \theta_{j k}(z), \\
\mu(z)^{2 j+k+3} \chi_{j k}(z)=\chi_{j k}^{\prime}\left(z^{\prime}\right)=\left|\begin{array}{cc}
\chi_{j}^{\prime}(z) & \theta_{k}^{\prime}\left(z^{\prime}\right) \\
\frac{-1}{4 j+4} \frac{d}{d z^{\prime}} \chi_{j}^{\prime}\left(z^{\prime}\right) & \frac{-1}{2 k} \frac{d}{d z^{\prime}} \theta_{k}^{\prime}\left(z^{\prime}\right)
\end{array}\right| \\
=\mu(z)^{2 j+k+2} \frac{d z}{d z^{\prime}} \chi_{j k}(z) .
\end{gathered}
$$

If $\left(\cdots \theta_{j k}, \cdots, \chi_{j k} \cdots\right) \neq \equiv(0, \cdots, 0)$ then we have

$$
\mu(z) \frac{d z^{\prime}}{d z} \equiv 1
$$

and

$$
\begin{aligned}
\theta_{j}^{\prime}\left(z^{\prime}\right)\left(d z^{\prime}\right)^{j} & =\theta_{j}(z)\left(\mu(z) d z^{\prime}\right)^{j}=\theta_{j}(z)(d z)^{j}, \\
\chi_{j}^{\prime}\left(z^{\prime}\right)\left(d z^{\prime}\right)^{2 j+2} & =\chi_{j}(z)\left(\mu(z) d z^{\prime}\right)^{2 j+2}=\chi_{j}(z)(d z)^{2 j+2} .
\end{aligned}
$$

By virtue of (4) this shows

$$
\left\{\theta_{j}(z)(d z)^{j}, d z\right\}=\left\{\theta_{j}(z)(d z)^{j}, d z^{\prime}\right\} \quad(3 \leq j \leq n),
$$

and thus $z^{\prime}$ is a Möbius transformation of $z$. Namely $L_{n}\left(Q^{\prime} \mid z^{\prime}, y\right)$ is equivalent to $L_{n}(Q \mid z, y)$. Assume that $\left(\cdots, \theta_{j k}, \cdots, \chi_{j, k}, \cdots\right)=(0, \cdots 0)$. Then by virtue of Lemma 2 there exist a constant $c$ and $a$ function $u(z)$ such that

$$
\begin{aligned}
\theta_{j}(z) & =c_{j}\left(\frac{d u}{d z}\right)^{j} \\
\chi_{j}(z) & =\frac{c_{j}^{2}(d u / d z)^{2 j}}{2(2 j+1)}\{u, z\}=\frac{-c_{j}^{2}(d u / d z)^{2 j}}{2(2 j+1)}\{z, u\}, \\
\chi_{j k}(z) & =\left|\begin{array}{cc}
\chi_{j} & \theta_{k} \\
\frac{-1}{4 j+4} \frac{d \chi_{j}}{d z} & \frac{-1}{2 k} \frac{d \theta_{k}}{d z}
\end{array}\right| \\
& =\frac{-c_{j}^{2}(d u / d z)^{2 j+2}}{4(2 j+1)(2 j+2)} \frac{d}{d z}\{z, u\}=0
\end{aligned}
$$

This shows $\{z, u\}=-\frac{\alpha}{2}$ with $\alpha$ in $C$, and thus

$$
\begin{aligned}
& \left(\cdots \theta_{j}(z), \cdots, \theta_{j k}(z), \cdots, \chi_{j}(z), \cdots, \chi_{j k}(z), \cdots\right) \\
& \quad=\left(\cdots, c_{j}, \cdots, 0 \cdots, \frac{-c_{j}^{2} \alpha}{4(2 j+1)}, \cdots 0 \cdots 0\right) .
\end{aligned}
$$


Hence $\tilde{C}$ is a point. We may assume $z=e^{\alpha u}$ within Möbius transformation. This means

$$
\begin{gathered}
\frac{d u}{\alpha z}=\frac{1}{\alpha z}, \quad \theta_{j}(z)=c_{j}(\alpha z)^{-j} \\
z^{-n} L_{n}(Q \mid z, y)=\sum\left(\begin{array}{c}
n \\
l
\end{array}\right) \gamma_{l}\left(\frac{1}{z} \frac{d}{d z}\right)^{n-l} y
\end{gathered}
$$

with $\gamma_{3}, \cdots, \gamma_{n}$ in $C$. The fundamental solution of this type of linear differential operator is given by

$$
\left(z^{\lambda_{1}}, z^{\lambda_{1}} \log z, \cdots, z^{\lambda_{1}}(\log z)^{m_{1}}, \cdots, z^{\lambda_{r}}, z^{\lambda_{r}} \log z, \cdots, z^{\lambda_{r}}(\log z)^{m_{r}}\right) .
$$

\section{Coordinate-free formulation}

We shall reformulate the above results in terms of bundles. Let $u$ be an independent variable, and let

$$
d^{l} u \quad(l=1,2,3, \cdots)
$$

be independent variables over ring $C\{u\}$ of convergent power series in $u$. A derivation $d$ is defined in polynomial algebra

$$
C\{u\}\left[\cdots, d^{l} u, \cdots\right]
$$

as follows

$$
\begin{gathered}
d f=\frac{d f}{d u} d u \quad(f \in C\{u\}), \\
d\left(d^{l} u\right)=d^{l+1} u \quad(l=1,2,3, \cdots) .
\end{gathered}
$$

Wronskian of $\left(\varphi_{1}(u), \cdots, \varphi_{n}(u)\right)$ is defined by

$$
W_{\left(\varphi_{1}, \cdots, \varphi_{n}\right)}=\left|\begin{array}{ccc}
\varphi_{1}, & \cdots, & \varphi_{n} \\
d \varphi_{1}, & \cdots, & d \varphi_{n} \\
\vdots & & \vdots \\
d^{n-1} \varphi_{1}, & \cdots, & d^{n-1} \varphi_{n}
\end{array}\right|
$$

The relation between this Wronskian and the usual one is given by 


$$
W_{\left(\varphi_{1}, \cdots, \varphi_{n}\right)}=\left|\begin{array}{ccc}
\varphi_{1}, & \cdots, & \varphi_{n} \\
\frac{d}{d u} \varphi_{1}, & \cdots, & \frac{d}{d u} \varphi_{n} \\
\vdots & & \vdots \\
\left(\frac{d}{d u}\right)^{n-1} \varphi_{1}, \cdots, & \left(\frac{d}{d u}\right)^{n-1} \varphi_{n}
\end{array}\right|(d u)^{n(n-1) / 2} .
$$

We denote

$$
\begin{aligned}
\tilde{L}_{n}(\tilde{p} \mid y) & =d^{n} y+\sum_{l=1}^{n}\left(\begin{array}{c}
n \\
l
\end{array}\right) \tilde{p}_{l}\left(\cdots, d^{n} \varphi_{g}, \cdots\right) d^{n-l} y \\
& =(-1)^{n} W_{\left(\varphi_{1}, \cdots, \varphi_{n}\right)}^{-1}\left|\begin{array}{cccc}
y, & \varphi_{1}, & \cdots, \varphi_{n} \\
d y, & d \varphi_{1}, & \cdots, & d \varphi_{n} \\
\vdots & \vdots & \vdots \\
d^{n} y, & d^{n} \varphi_{1}, \cdots, d^{n} \varphi_{n}
\end{array}\right|,
\end{aligned}
$$

then

$$
L_{n}(\tilde{p} \mid y)=L_{n}(p \mid u, y)(d u)^{n},
$$

$\tilde{L}_{n}(\tilde{p} \mid y)\left(L_{n}(p \mid u, y)\right)$ is called to be semi-canonical if $\tilde{p}_{1} \equiv 0 . \quad \tilde{L}_{n}(\tilde{p} \mid u, y)$ is semi-canonical if and only if

$$
W_{\left(\varphi_{1}, \cdots, \varphi_{n}\right)} \equiv \gamma(d u)^{n}
$$

with $\gamma \neq 0$ in $C$.

Changing the dependent variable

$$
y \mapsto \lambda(u) y
$$

with a suitable $\lambda(u)(\lambda(0) \neq 0)$, we may transform any $\tilde{L}_{n}(\tilde{p} \mid u, y)$ to a unique semi-canonical form.

A differential invariant of weight $p$ of $\tilde{L}_{n}(\tilde{p} \mid u, y)$ is defined by

$$
\Phi\left(\cdots,\left(\frac{d}{d u}\right)^{l} p_{j}, \cdots\right)(d u)^{m}
$$

with a differential invariant of weight $m$

$$
\Phi\left(\cdots,\left(\frac{d}{d u}\right)^{l} p_{j}, \cdots\right) .
$$

For a semi-canonical form $\tilde{L}_{n}(\tilde{p} \mid y)$ the set of differential invariants of weight $m$ coincides with the set of differential polynomial with coeffi- 
cients in $\boldsymbol{C}$

$$
\Phi\left(\cdots, d^{l} \tilde{p}_{j}, \cdots\right)
$$

such that

$$
\Phi\left(\cdots, d^{l} \tilde{p}_{j}, \cdots\right)=\Phi\left(\cdots,\left(\frac{d}{d u}\right)^{l} p_{j}, \cdots\right)(d u)^{m}
$$

For each differential invariant $\Phi$ of weight $m$ of $L_{n}(p \mid u, y)$ we denote

$$
\tilde{\Phi}=\Phi\left(\cdots, d^{l} \tilde{p}_{j}, \cdots\right)=\Phi\left(\cdots,\left(\frac{d}{d u}\right)^{l} p_{j}, \cdots\right)(d u)^{m},
$$

then we get the system of fundamental differential invariants

$$
\left(\tilde{\theta}_{3}, \cdots, \tilde{\theta}_{n}\right)
$$

of $\tilde{L}_{n}(\tilde{p} \mid y)$. Moreover the system of differential invariants

$$
\left(\tilde{\theta}_{3}, \cdots, \tilde{\theta}_{n}, \cdots, \tilde{\theta}_{j k}, \cdots, \tilde{\chi}_{j}, \cdots, \tilde{\chi}_{j k}, \cdots\right)
$$

corresponding to

$$
\left(\theta_{3}, \cdots, \theta_{n}, \cdots, \theta_{j k}, \cdots, \chi_{j}, \cdots, \chi_{j k}, \cdots\right) .
$$

\section{Several variable case}

Let $u_{1}, \cdots, u_{r}$ be independent variables and let

$$
d^{l} u_{j} \quad(l=1,2,3, \cdots ; j=1,2, \cdots, r)
$$

be independent variables over ring of convergent power series $C\left\{u_{1}, \cdots, u_{r}\right\}$. We define a derivation $d$ on commutative polynomial algebra

$$
C\left\{u_{1}, \cdots, u_{r}\right\}\left[\cdots, d^{l} u_{j}, \cdots\right]
$$

over $C\left\{u_{1}, \cdots, u_{r}\right\}$ as follows

$$
\begin{array}{ll}
d f=\sum_{j=1}^{r} \frac{\partial f}{\partial u_{j}} d u_{j} & \left(f \in C\left\{u_{1}, \cdots, u_{r}\right\}\right), \\
d\left(d^{l} u_{j}\right)=d^{l+1} u_{j} & (l=1,2,3, \cdots ; j=1,2, \cdots, r) .
\end{array}
$$

Remark. For any holomorphic curve

$$
t \mapsto\left(u_{1}(t), \cdots, u_{r}(t)\right)
$$

we can associate a differential algebra homomorphism 


$$
C\left\{u_{1}, \cdots, u_{r}\right\}\left[\cdots, d^{l} u_{j}, \cdots\right] \rightarrow C\{t\}\left[\cdots, d^{l} t, \cdots\right]
$$

such that

$$
d^{l} \varphi\left(u_{1}, \cdots, u_{r}\right) \mapsto d^{l} \varphi\left(u_{1}(t), \cdots, u_{r}(t)\right) .
$$

For a system $\left(\varphi_{1}\left(u_{1}, \cdots, u_{r}\right), \cdots, \varphi_{n}\left(u_{1}, \cdots, u_{r}\right)\right)$ Wronskian of $\left(\varphi_{1}, \cdots\right.$, $\left.\varphi_{n}\right)$ is defined by

$$
W_{\left(\varphi_{1}, \cdots, \varphi_{n}\right)}=\left|\begin{array}{ccc}
\varphi_{1}, & \cdots, & \varphi_{n} \\
d \varphi_{1}, & \cdots, & d \varphi_{n} \\
\vdots & & \vdots \\
d^{n-1} \varphi_{1}, & \cdots, & d^{n-1} \varphi_{n}
\end{array}\right|
$$

We denote

(6)

$$
\begin{aligned}
\tilde{L}_{n}(\tilde{p} \mid y) & =(-1)^{n} W_{\left(\varphi_{1}, \cdots, \varphi_{n}\right)}^{-1}\left|\begin{array}{cccc}
y, & \varphi_{1}, & \cdots, & \varphi_{n} \\
d y, & d \varphi_{1}, & \cdots, & d \varphi_{n} \\
\vdots & \vdots & \vdots \\
d^{n} y, & d^{n} \varphi_{1}, \cdots, d^{n} \varphi_{n}
\end{array}\right| \\
& =d^{n} y+\sum_{l=1}^{n}\left(\begin{array}{c}
n \\
l
\end{array}\right) \tilde{p}_{l}\left(\cdots, d^{l} \varphi, \cdots\right) d^{n-l} y .
\end{aligned}
$$

We may define differential invariants of weight $p$ of $\tilde{L}_{n}(p \mid y)$ by the same differential polynomials as differential invariants of one variable case.

\section{REFERENCES}

[1] H. Morikawa, Invariant Theory (in Japanese), Kinokuniya, Tokyo 1977.

[2] - Some geometric and analytic applications of invariant theoretic method, to appear in Nagoya Math. J., 80 (1980).

[ 3 ] Wilczinski, Projective Differential Geometry of Curves and Ruled Surfaces, Chelsea 1961. 